\title{
Necking Modes in Multilayers and their Influence on Tearing Toughness
}

\section{Citation}

Hutchinson, John W. 2013. Necking modes in multilayers and their influence on tearing toughness. Mathematics and Mechanics of Solids 19, no. 1: 39-55.

\section{Published Version}

doi:10.1177/1081286513505468

\section{Permanent link}

http://nrs.harvard.edu/urn-3:HUL.InstRepos:11643695

\section{Terms of Use}

This article was downloaded from Harvard University's DASH repository, and is made available under the terms and conditions applicable to Open Access Policy Articles, as set forth at http:// nrs.harvard.edu/urn-3:HUL.InstRepos:dash.current.terms-of-use\#OAP

\section{Share Your Story}

The Harvard community has made this article openly available.

Please share how this access benefits you. Submit a story.

Accessibility 
Published in Mathematics and Mechanics of Solids 2014, Vol 19(1) 39-55.

\title{
Necking modes in multilayers and their influence on tearing toughness
}

\author{
John W. Hutchinson \\ School of Engineering and Applied Sciences, Harvard University \\ Cambridge, MA 02138, USA
}

\begin{abstract}
Periodic bifurcation modes that occur in ductile multilayered plates or sheets stretched in plane strain tension are analyzed to reveal whether necks are likely to localize at the scale of the thickness of individual layers or at the scale of the full thickness of the multilayer. The energy dissipated in tearing a ductile multilayer scales with the extent of the localized thinning region in the tensile direction. If plates or sheets with high tearing toughness are desired, the combination of layers should be chosen to suppress necking localization at the scale of individual layers. Insight into the properties and thicknesses of the layers required to suppress short wavelength necking is revealed by a bifurcation analysis of multilayers comprised of metal layers having different strength and hardening behaviors and multilayers combining metal and elastomer layers. Several examples suggest that when localization takes place at the scale of the individual layers it may occur in the form of a band inclined through the thickness.
\end{abstract}

\section{Keywords}

Layered materials, necking, bifurcation, power law materials, neo-Hookean materials

\section{Introduction}

Sheets and plates with specific performance objectives are increasingly multilayers comprised of different material layers bonded together. GLARE, for example, is a laminate combining layers of glass fiber in epoxy with layers of aluminum to achieve enhanced fatigue and tearing strength. Many packaging materials are laminates of metals and polymers designed for multiple objectives including tearing resistance. Flexible electronic systems employ thin 
metal films on polymer or elastomer substrates which allow the films to be stretched beyond the limit a free-standing film can sustain.

A tensile tearing test on a multilayer plate or sheet can be carried out by introducing a long central crack then subjecting the plate to tension perpendicular to the crack. If the multilayer is ductile, a necking zone extends outward from the tip of the crack. At some point, as the overall tension is increased, this localized necking zone begins to undergo separation starting at the crack tip. With increasing overall tensile stretching, tearing spreads from the initial crack preceded by a zone of necking. The mechanics of the combination of the crack and the extended necking zone is covered by the class of models proposed by Barenblatt [1] and Dugdale [2]. For ductile monolithic metal plates or sheets, most of the tearing energy is dissipated by plastic deformation in the necked region that advances ahead of the tear [3]. Moreover, because the height of the neck scales with the plate thickness, the tearing toughness, which is the energy required to tear a unit cross-sectional area of plate measured in $\mathrm{Jm}^{-2}$, scales with the thickness of the plate or sheet.

The implications of this scaling are significant as the following simple example illustrates. A single plate of thickness $2 h$ has approximately twice the tearing toughness of two stacked, but unbonded, plates of the same material, each with thickness $h$. While the strength of these two systems is nominally the same the single plate has twice the tearing toughness because the height of the neck is twice as large as that of the two-layer plate. Now suppose a multi-layer is formed by including a thin core layer sandwiched between and bonded to each of the two stacked plates. Assume the core material is relative light and that its strength is low compared to the outer plates such that the strength of the multilayered plate is essentially the same as the original stacked plates. What about tearing toughness of the multilayer? If the core layer is able suppress localized necking in each of the outer plates and force the height of the neck to scale with the total thickness of the multi-layer, then the tearing toughness of the multilayer will be comparable to, or perhaps even larger than, that of the single layer plate with thickness $2 h$.

This example motivates the study in this paper by emphasizing the importance for multilayers of whether necking occurs at the scale of the individual layers or at the scale of the total thickness of the multilayer. The method employed in this study is a bifurcation analysis of infinitely long multilayers examining the dependence of the bifurcation strain on modal 
wavelength. Further work based on post-bifurcation finite element calculations will be required to fully reveal the localization process, as will be emphasized in the Discussion.

\section{Necking bifurcation problem for a multilayer in plane strain tension}

The multilayer under consideration is shown in Fig. 1. The multilayer is taken to be infinite in the $x_{1}$ direction and periodic modes in this direction will be sought. The layering, materials and the layer thicknesses, will be taken to be symmetric with respect to the centerline, $x_{2}=0$. For analysis, this allows attention to be focused on the upper half of the multilayer, and for this purpose the layers are numbered starting with $i=1$ for the center layer up to $i=M$ for the top layer, with the total number of layers being $2 M-1$. The thickness of the $i$ th layer at the point of bifurcation is denoted by $h_{i}, i=1, M$, and the total thickness of the of all $2 M-1$ layers of multilayer at bifurcation is $H$. The layers remain fully bonded and the top and bottom surfaces of the multilayer are traction free. Combinations of two materials will be considered with material A always at the center and material B alternating with material A in the outer layers.

The analysis which follows draws heavily on the plane strain bifurcation analysis of a single layer in tension presented by Hill and Hutchinson [4], which in turn draws on earlier work of Biot [5]. For the most part the notation use here is also the same as that in [4]. In the undeformed state the layers are assumed to be unstressed. Under plane strain constraint, i.e., no straining in the $x_{3}$ direction, the multilayer is subject to an overall tensile stretch, $\lambda$, and logarithmic tensile strain, $\varepsilon=\ln \lambda$, Prior to bifurcation, each layer is subject to a uniform true stress, $\sigma_{11}=\sigma$, which varies from layer to layer. The out-of-plane stress, $\sigma_{33}$, will not directly enter into the analysis.

In this paper, the specific materials considered are incompressible, isotropic and nonlinear elastic. In addition, the strain energy/volume of the materials considered here are characterized by a single deformation measure which in plane strain tension can be represented as $W(\varepsilon)$ with $\varepsilon=\ln (\lambda)$. Plastic materials will be modeled by finite strain $J_{2}$ deformation 
theory which falls into this category. In the state of plane strain tension with $\sigma_{11}=\sigma$, the constitutive relation for plane strain increments is $[4,5]$

$$
\hat{\sigma}_{11}-\hat{\sigma}_{22}=\left(E_{t} / 2\right)\left(D_{11}-D_{22}\right), \hat{\sigma}_{12}=2 \mu D_{12} \text { with } D_{11}+D_{22}=0
$$

where $\hat{\sigma}$ denotes the Jaumann stress rate and $D_{i j}$ is the Eulerian strain rate. In an increment of plane strain tension with $\hat{\sigma}_{22}=0, \hat{\sigma}_{11}=E_{t} D_{11}$, and thus $E_{t}$ is the tangent modulus of the true stress-logarithmic strain curve in plane strain tension at $\sigma$. (In [4], the notation $4 \mu^{*}$ was used for $E_{t}$.) The other incremental modulus, $\mu$, governs in-plane shearing. In plane strain tension for materials characterized by the strain energy density $W(\varepsilon)[4]$ :

$$
\sigma=\frac{d W}{d \varepsilon}, \quad E_{t}=\frac{d \sigma}{d \varepsilon}, 2 \mu=\frac{\lambda^{4}+1}{\lambda^{4}-1} \sigma=\operatorname{coth}(2 \varepsilon) \sigma
$$

One class of materials considered is a pure power-law hardening, finite strain $J_{2}$ deformation theory solid with hardening exponent $N$ [6]. In plane strain tension, $W=\sigma_{0} \varepsilon^{N+1} /(N+1)$, and (2) give

$$
\sigma=\sigma_{0} \varepsilon^{N}, E_{t}=N \sigma_{0} \varepsilon^{N-1}, 2 \mu=\sigma_{0} \operatorname{coth}(2 \varepsilon) \varepsilon^{N}
$$

The second class of materials is neo-Hookean materials which will be layered in combination with power law materials. These have $W=\mu_{0}\left(\lambda^{2}+\lambda^{-2}-2\right)=2 \mu_{0}(\cosh (2 \varepsilon)-1)$ and

$$
\sigma=2 \mu_{0} \sinh (2 \varepsilon), \frac{E_{t}}{4}=\mu=\mu_{0} \cosh (2 \varepsilon)
$$

The ground state shear modulus, $\mu_{0}$, is the sole material parameter.

The derivation of the equations governing the bifurcation problem closely follows that given in [4] and the reader is referred to that reference for some of the details. Cartesian coordinates are used and the reference state is the fundamental solution at the point of bifurcation when each layer is in a state of uniform plane strain tension. With $\left(v_{1}, v_{2}\right)$ as the velocity components (increments of displacement) associated with the bifurcation mode:

$$
D_{11}=v_{1,1}, \quad D_{22}=v_{2,2}, \quad 2 D_{12}=2 D_{21}=v_{1,2}+v_{2,1}
$$

Incompressibility allows one to introduce a velocity potential, $\psi\left(x_{1}, x_{2}\right)$, such that 


$$
v_{1}=\psi_{, 2}, \quad v_{2}=-\psi_{, 1}
$$

Nominal stress rates are related to the Juamann rates by

$$
\left.\begin{array}{l}
\dot{n}_{11}=\hat{\sigma}_{11}-\sigma v_{1,1}, \dot{n}_{22}=\hat{\sigma}_{22} \\
\dot{n}_{12}=\hat{\sigma}_{12}+\sigma\left(v_{2,1}-v_{1,2}\right), \dot{n}_{21}=\hat{\sigma}_{12}-\sigma\left(v_{2,1}+v_{1,2}\right)
\end{array}\right\}
$$

These rates are defined such that $\dot{n}_{i j} d A$ is the force increment in the $x_{j}$ direction on a material surface element with area $d A$ aligned perpendicular to the $x_{i}$ direction in the state just prior to the current increment.

Equations for incremental equilibrium are listed in [4] leading to the governing bifurcation equations. Alternatively, one can also obtain the equations using the following variational approach which was also given in [4,5]. The quadratic functional of the velocity potential which governs the bifurcation problem [7] is

$$
\begin{aligned}
& \Phi=\int_{S} U d S \\
& U=\frac{1}{2} \dot{n}_{i j} v_{i, i}=\frac{1}{2}\left(\left(E_{t}-\sigma\right) \psi_{, 12}{ }^{2}+\sigma \psi_{, 11}{ }^{2}+(\mu-\sigma / 2)\left(\psi_{, 11}-\psi_{, 22}\right)^{2}\right)
\end{aligned}
$$

Here, $S$ is the area covering the full thickness of the multilayer for one period, $L$, of the mode in the current fundamental state and $\left(\sigma, E_{t}, \mu\right)$ vary from layer to layer but are uniform within each layer. With $L$ specified, $\Phi>0$ for all admissible velocities at overall strains, $\varepsilon$, below the lowest bifurcation strain. At the lowest bifurcation strain, $\Phi=0$ when evaluated in terms of the bifurcation mode, and the first variation of $\Phi$ vanishes with respect to admissible variations of $\psi$. Vanishing of the first variation requires the following partial differential equation to be satisfied in each layer

$$
(\mu+\sigma / 2) \psi_{, 1111}+\left(E_{t}-2 \mu\right) \psi_{, 1122}+(\mu-\sigma / 2) \psi_{, 2222}=0
$$

Conditions for continuity of velocities, $\left(v_{2} \cdot v_{1}\right)$, and traction rates, $\left(\dot{n}_{22}, \dot{n}_{21}\right)$, across an interface between two layers can be expressed as (in the order listed) 


$$
[\psi]=0,\left[\psi_{, 2}\right]=0,\left[p \psi_{, 112}+q \psi_{, 222}\right]=0,\left[q\left(\psi_{, 11}-\psi_{, 22}\right)\right]=0
$$

where [ ] denotes a value evaluated just above the interface minus that just below the interface, $p=\left(E_{t}-\mu-\sigma / 2\right)$ and $q=(\mu-\sigma / 2)$. On the upper free surface of the top layer the traction free condition requires

$$
p \psi_{, 112}+q \psi_{, 222}=0, q\left(\psi_{, 11}-\psi_{, 22}\right)=0
$$

The symmetry of the multilayer in the fundamental state allows consideration of bifurcation modes that are either symmetric or anti-symmetric with respect to the centerline such that

$$
\left.\begin{array}{l}
\psi=0, \quad \psi_{, 22}=0 \quad \begin{array}{l}
\text { (symmetric modes) } \\
\psi_{, 2}=0, \psi_{, 222}=0
\end{array} \\
\text { (anti-symmetric modes) }
\end{array}\right\} \quad \text { on } x_{2}=0
$$

\section{The Considère strain for the multilayer and requirements for ellipticity}

With each layer in plane strain tension, the Considère strain, $\varepsilon_{C}$, is the overall uniform strain imposed on the multilayer at which the maximum load is attained, assuming a maximum exists. A long multilayer stretched beyond this strain will necessarily begin to undergo a necking localization somewhere along its neck according to the well-known reasoning of Considère. It is readily shown that the Considère condition for the multilayer has the same form as that for a single layer,

$$
\bar{\sigma}=\bar{E}_{t}
$$

but expressed in terms of the average true stress and tangent modulus

$$
\bar{\sigma}=H^{-1} \int_{-H / 2}^{H / 2} \sigma d x_{2} \text { and } \bar{E}_{t}=H^{-1} \int_{-H / 2}^{H / 2} E_{t} d x_{2}
$$

where $H$ is the total thickness of the multilayer in the current pre-bifurcated state.

In [4] it was proved that bifurcation for a single layer in plane strain tension in a mode of any wavelength is bounded from below by the Considère strain, as long as the side condition $\mu>\sigma / 2$ is met. For a multi-layer, bifurcation in a short wavelength mode can occur below the 
Considère strain, as will be illustrated in the paper. In all cases, bifurcation strain associated with the long wavelength limit, $L \rightarrow \infty$, is the Considère strain if it exists.

In this paper attention has been restricted to examples such the parameters $\left(\sigma, E_{t}, \mu\right)$ in each layer ensure that the pde (10) is elliptic, i.e., has no real characteristics, up to and including lowest bifurcation point for each period considered. Ellipticity ensures the smooth solutions within each layer listed in the next section. A full discussion of whether (10) is elliptic, hyperbolic or parabolic is given in [4]. In addition to $\mu>\sigma / 2$, ellipticity requires

$$
E_{t}>2 \mu\left(1-\sqrt{1-(\sigma / 2 \mu)^{2}}\right)
$$

In the examples analyzed in this paper, based on either (3) or (4), the side condition $\mu>\sigma / 2$ is satisfied at all tensile strains. Condition (15) is always met for the neo-Hookean material (4). For the power-law material (3) in plane strain tension, (15) reduces to

$$
(\varepsilon / \sinh (2 \varepsilon))(\cosh (2 \varepsilon)-1)<N
$$

The strain at which the equality is achieved in (16), corresponding the loss of ellipticity, is plotted in Fig. 2. It is considerably larger than the Considère strain, $\varepsilon_{C}=N$, for a single, freestanding layer of the same material. However, when layers with different hardening exponents are combined the ellipticity limit may be reached in a layer with the lowest exponent before the Considère strain of the multilayer is reached. The ellipticity range of the incremental equations for the power-law material under general strain states has been presented in [6].

\section{Solution for the bifurcation strain and mode}

Bifurcation solutions for the multilayer are sought with period $L$ referred to the state at bifurcation in the form

$$
\psi=f\left(x_{2}\right) \sin \left(2 \pi x_{1} / L\right) \Rightarrow v_{1}=f^{\prime} \sin \left(2 \pi x_{1} / L\right), v_{2}=-(2 \pi / L) f \cos \left(2 \pi x_{1} / L\right)
$$

with ()$^{\prime}=d() / d x_{2}$. Eq. (10) separates providing the following ode for $f\left(x_{2}\right)$ : 


$$
\left(\mu-\frac{\sigma}{2}\right) f^{\prime \prime \prime \prime}-\left(E_{t}-2 \mu\right)\left(\frac{2 \pi}{L}\right)^{2} f^{\prime \prime}+\left(\mu+\frac{\sigma}{2}\right)\left(\frac{2 \pi}{L}\right)^{4} f=0
$$

where the coefficients are piecewise constant within each layer.

\subsection{The general solution within any layer}

Let $R \equiv\left(2 \mu-E_{t}\right)^{2}-\left(4 \mu^{2}-\sigma^{2}\right)$. If $\mu>\sigma / 2$ and the ellipticity condition (15) holds, the general solution to (18) within any layer is given by the following. Let $y=x_{2}-\bar{x}_{2}$, where $x_{2}=\bar{x}_{2}$ at the bottom of the layer. If $R>0$,

$$
f=c_{1} \cosh (2 \pi \alpha y / L)+c_{2} \sinh (2 \pi \alpha y / L)+c_{3} \cosh (2 \pi \beta y / L)+c_{4} \sinh (2 \pi \beta y / L)
$$

with

$$
\alpha=\sqrt{\frac{E_{t}-2 \mu+\sqrt{R}}{2 \mu-\sigma}}, \quad \beta=\sqrt{\frac{E_{t}-2 \mu-\sqrt{R}}{2 \mu-\sigma}}
$$

If $R<0$,

$$
\begin{aligned}
f= & c_{1} \cosh (2 \pi \alpha y / L) \cos (2 \pi \beta y / L)+c_{2} \cosh (2 \pi \alpha y / L) \sin (2 \pi \beta y / L) \\
& +c_{3} \sinh (2 \pi \alpha y / L) \sin (2 \pi \beta y / L)+c_{4} \sinh (2 \pi \alpha y / L) \cos (2 \pi \beta y / L)
\end{aligned}
$$

with

$$
\alpha+i \beta=\sqrt{\frac{E_{t}-2 \mu+i \sqrt{-R}}{2 \mu-\sigma}}
$$

where $i=\sqrt{-1}$ and the square root lies in the $1^{\text {st }}$ quadrant of the complex plane with $\alpha>0$ and $\beta>0$. For the power-law material (3), $R \leq 0$ with $R=0$ at the ellipticity limit (16). For the neo-Hookean material, $R=\sigma>0$.

At the bottom of the layer, the quantities required for continuity of velocities and nominal traction rates in (11) are 


$$
\left(\psi, \psi_{, 2}, p \psi_{, 112}+\psi_{, 222}, q\left(\psi_{, 11}-\psi_{, 22}\right)\right)=a \sin \left(2 \pi x_{1} / L\right)
$$

where $a$ is the 4-vector $\left(a_{1}, a_{2}, a_{3}, a_{4}\right)$ given by $a_{i}=B_{i j} c_{j}$, with $c=\left(c_{1}, c_{2}, c_{3}, c_{4}\right)$ and $B$ is the $4 \times 4$ matrix given in Appendix. The same set of quantities defined in (21) evaluated at the top of the layer are given by $a_{i}=T_{i j} c_{j}$ where $T$ is also given in Appendix.

\subsection{Conditions at the free surface of the top layer and at the centerline of the multilayer}

With $T$ and $c$ representing the top layer (the $M^{\text {th }}$ layer), conditions (12) for a tractionfree surface are

$$
T_{3 j} C_{j}=0 \& T_{4 j} C_{j}=0 \text { or } C^{(M)} C=0
$$

where $C^{(M)}$ is the $2 \times 4$ matrix with components $C_{1 j}^{(M)}=T_{3 j}$ and $C_{2 j}^{(M)}=T_{4 j}$.

The $1^{\text {st }}$ layer containing the centerline is treated differently. For this layer, $y=0$ is taken as the centerline in expressions (19) and (20). Then, with $c$ representing the $1^{\text {st }}$ layer, (13) requires

$$
\begin{array}{ll}
c_{1}=c_{3}=0 & \text { (symmetric modes) } \\
c_{2}=c_{4}=0 & \text { (anti-symmetric modes) }
\end{array}
$$

For the symmetric modes, let $c^{(1)}=\left(c_{2}, c_{4}\right)$ and, for the anti-symmetric modes, let $c^{(1)}=\left(c_{1}, c_{3}\right)$. Let $T$ be the $4 \times 4$ matrix evaluated at the top of the $1^{\text {st }}$ layer. The vector $a$ evaluated at the top of the $1^{\text {st }}$ layer is given by $a=T^{(1)} c^{(1)}$ where $T^{(1)}$ is the $4 \times 2$ matrix defined by

$$
\begin{aligned}
& T_{i 1}^{(1)}=T_{i 2}, T_{i 2}^{(1)}=T_{i 4} \quad \text { (symmetric modes) } \\
& T_{i 1}^{(1)}=T_{i 1}, T_{i 2}^{(1)}=T_{i 3} \quad \text { (anti-symmetric modes) }
\end{aligned}
$$

\subsection{The bifurcation condition and the bifurcation mode}

For each of the $M$ layers above the centerline (c.f., Fig. 1) other than the $1^{\text {st }}$ layer, denote the 4 -vectors and $4 \times 4$ matrix quantities defined above by $c^{(i)}, B^{(i)}, T^{(i)}(i=2, M)$. Using 
continuity of the $a$ vector across each interface, one can solve for $c^{(i)}$ sequentially in terms of $c^{(1)}$ with the result $c^{(i)}=D^{(i)} c^{(1)}$ where $D^{(i)}$ is the $4 \times 2$ matrix given by

$$
D^{(i)}=B^{(i)-1} T^{(i-1)} \ldots . . . B^{(3)-1} T^{(2)} B^{(2)-1} T^{(1)}, i=2, M
$$

The free-surface condition (22) provides the bifurcation equation

$$
A c^{(1)}=0
$$

with $A=C^{(M)} D^{(M)}$ as a $2 \times 2$ matrix. For a given period, $L$, the bifurcation strain is the overall strain at which $|A|=0$ is first attained as the strain is increased from zero, with both symmetric and anti-symmetric modes in contention. Note that $A$ depends on the symmetry condition through the $4 \times 2$ matrix $T^{(1)}$. At bifurcation, (26) provides a single amplitude factor for the mode because one of the two components of $c^{(1)}$ can be expressed in terms of the other, e.g., $A_{11} c_{1}^{(1)}+A_{12} c_{2}^{(1)}=0$. The coefficients $c$ for each layer can then be solved sequentially in terms of $c^{(1)}$ using (25) generating the full mode, which can be normalized as desired.

\section{The role of variations in layer strength on necking of multilayered power-law materials}

Even though attention will be restricted to materials characterized either by the powerlaw deformation theory or by a neo-Hookean solid, there is a rich set of possible effects. We attempt to reveal some of these effects as systematically as possible, including considerations related to the number and relative thickness of the layers. In this section attention is focused on multilayers comprised of layers of the power-law material (3) all having the same hardening exponent, $N$, but with the strength, $\sigma_{0}$, varying from layer to layer. In the following sections, multilayers with different hardening exponents will be considered, as will multilayers comprised of alternating layers of a power-law material and a neo-Hookean material.

With the power-law material (3) representing a simplified description of metal plasticity ${ }^{1}$, the multilayer in Fig. 1 is comprised of two alternating materials A and B, with

\footnotetext{
${ }^{1}$ An extensive literature exists regarding the use of nonlinear elastic models of plasticity, e.g., the $\mathrm{J}_{2}$ deformation theory, to characterize plasticity for buckling and bifurcation studies [8]. The applicability of deformation theory for these purposes will not be reviewed here except to note that bifurcation predictions based on deformation theory
} 


$$
\sigma=\sigma_{0}^{A} \varepsilon^{N_{A}} \quad(\text { material A }), \quad \sigma=\sigma_{0}^{B} \varepsilon^{N_{B}} \quad(\text { material B })
$$

In this section, identical hardening exponents, $N_{A}=N_{B} \equiv N$ will be consider such that the Considère condition for the maximum load of the multilayer is always attained at $\varepsilon_{C}=N$, as can be easily established. The central layer, layer 1, is taken to be material A with the sequential outer layers alternating between B and A. The main influence explored in this section is the role of the strength ratio of the two materials, $\sigma_{0}^{A} / \sigma_{0}^{B}$, with some consideration given to the number of layers and their arrangement.

The 3-layer multilayer ( $M=2$ ) will be considered first with material A in the central layer (layer 1) sandwiched between two outer layers of material B. At the point of bifurcation, the thickness of layer 1 is $h_{1}$ and the thickness of each of the two outer layers is $h_{2}$. The total thickness of the multilayer is $H=h_{1}+2 h_{2}$. For each normalized period, $L / H$, Fig. 3 presents the overall strain $\varepsilon$ at bifurcation for a three-layer with $h_{2} / h_{1}=1 / 2$, i.e., equal total thickness of materials $\mathrm{A}$ and $\mathrm{B}$, for a wide range of $\sigma_{0}^{A} / \sigma_{0}^{B}$ with $N=0.1$. In plane strain tension, the ratio of the layer thicknesses does not vary with strain and, thus, $2 h_{2} / h_{1}=1$ can also be regarded as the ratio prior to deformation. The scalloped nature of the curves in Fig. 3 reflects the switch from symmetric to anti-symmetric modes for as the period is varied. For all cases, the bifurcation strain approaches the Considère strain, $\varepsilon_{C}=N$, as $L / H$ becomes large, with the symmetric mode favored. Thus, a sufficiently long multilayer would begin to undergo necking in a long wavelength mode as soon as the Considère strain is exceeded. ${ }^{2}$ The present analysis supplies insight as to whether modes with considerably shorter wavelength are lurking at strains below, or just above, the Considère strain and are thus likely to play a role in the localization process at

generally are in closer accord with experiments than those based on incremental theories that employ a smooth yield surface, such as $\mathrm{J}_{2}$ flow theory. In the present context the difference is due to the fact that a smooth yield surface constrains $\mu$ to be the elastic shear modulus whereas the deformation theory gives a reduced value (3).

${ }^{2}$ The interpretation of the solution here is different from that in [4]. Here we imagine that the multilayer is very long compared to $H$ such that a bifurcation will always exist at the Considère strain in a long wavelength symmetric mode. In [4], the length of the layer was not necessarily assumed to be very long compared to its thickness and the periodic boundary conditions were interpreted as idealized boundary conditions (zero shear traction and constrained planar motion in the 1-direction) applied at the ends of a finite slab of length $L$. Here the focus is on whether short wavelength modes with lengths on the order of the individual layer thickness have bifurcation strains below the Considère strain, or slightly above the Considère strain, and are therefore 'in contention' in the localization process. 
overall strains slightly beyond the onset of long wavelength necking. It must be emphasized that the present analysis only provides some insight into whether short wavelength modes are likely to emerge - a full nonlinear post bifurcation analysis, probably requiring a finite element simulation, must be used to establish whether and how such modes emerge. The single material case, which has been well explored [9,10], suggests that the long-wavelength mode rapidly localizes to ongoing deformation confined to a region on the order of the thickness. For the multilayer the issue is whether a mode whose wavelength is long compared to the total thickness, $H$, will localize to a region on the order of $H$ in extent or to a shorter length set by the thickness of an individual layer. As noted in the Introduction, the lateral extent of the mode has significant implications for the tearing resistance of the multilayer.

For the case where a layer of the weak material is sandwiched between two layers of strong material, $\sigma_{A} / \sigma_{B}=0.05$ in Fig. $3 \mathrm{~b}$, the bifurcation strain associated with the short wavelength, anti-symmetric mode having $L / H=2$ is nearly the same as that of the long wavelength symmetric mode having $L / H=10$. The mode shapes for these two cases are plotted in Fig. 4. The short wavelength mode is expected to be in contention in the localization process, although this would have to be established by a post-bifurcation analysis. The local necks in the outer layers for $L / H=2$ in Fig. 4 are offset from one another in the lateral direction by a half wavelength. This suggests that localization would occur as an inclined band across the multilayer.

When the strong material is the central layer, e.g., $\sigma_{B} / \sigma_{A}=0.05$ in Fig. 3a, there are also shorter wavelength, symmetric modes at bifurcation strains only slightly above the Considère strain, as expected, because the in the limit as $\sigma_{B} / \sigma_{A} \rightarrow 0$, the result becomes that for a single layer of material A with half the total thickness $H$. The results in Fig. 3 also indicate that bifurcation in the short wavelength modes is well separated from that in the long wavelength modes when the strength of the weaker layers is more than, approximately, a quarter that of the stronger layers. However, a more precise evaluation of the transition ratio of layer strengths at which localization would switch from being set by the multilayer thickness to the individual layer thickness would require a full nonlinear analysis. 
The influence of the number of layers in a multilayer of alternating weak and strong layers is illustrated in Fig. 5 for both 5 and 9 equal-thickness layers. For each of these multilayers the middle (and outermost layers) are comprised of the strong material. The 5-layer case has three strong layers and two weak layers, while the 9-layer case has five strong layers and four weak layers. For comparison purposes, the bifurcation strain of a single layer having the same total thickness, $H$, is included - the material has the same hardening exponent as the layers in the multilayer and thus this curve applies for any strength $\sigma_{0}$. For the two multilayers, a local minimum in the bifurcation strain only slightly above the Considère strain occurs at in short wavelength mode with period which is roughly inversely proportional to the number of layers, i.e., the wavelength scales with the individual layer thickness. The corresponding mode shapes are shown in Fig. 5b. The existence of these low bifurcation strains results again suggest that localization might develop with a lateral scale on the order of the individual layer thickness, in accord with what would be expected for very weak intermediate layers. The thinnest regions in the strong layers are offset from one another by a half-period suggesting again that localization across the multilayer would occur as an inclined band of local necks. The bifurcation mode in the weaker layers in Fig. 5b involves very little change in layer thickness, and a close inspection of the bifurcation mode within these layers reveals that the bifurcation strains are dominantly shearing.

\section{The role of a soft, high hardening interlayer in the necking of multilayered power-law materials}

Consider a multilayer comprised of layers of power-law materials A and B defined in (3) with differing hardening exponents. Denote the ratio of the total thickness of the layers comprising material B to that for the layers comprising material A by $h_{B} / h_{A}$, and recall that this ratio remains constant under plane strain tension. Condition (14) for the Considère strain $\varepsilon_{C}$ of the multilayer is

$$
\varepsilon_{C}^{N_{A}+1}\left(1-N_{A} / \varepsilon_{C}\right)+r \varepsilon_{C}^{N_{B}+1}\left(1-N_{B} / \varepsilon_{C}\right)=0 \quad \text { with } \quad r=h_{B} \sigma_{0}^{B} / h_{A} \sigma_{0}^{A}
$$

A plot of the Considère strain for selected hardening exponents is presented in Fig. 6. 
Attention will be limited to a two cases: a 3-layer plate with two strong, low hardening layers (material B) separated by a single layer of a relatively weak, high hardening material (material A), and the reversed layering with the strong, low hardening material as the central layer. At issue is whether higher hardening of the weak central layer is able to suppress short wavelength modes. The bifurcation results in Fig. 7 have $h_{B} / h_{A}=1\left(h_{2} / h_{1}=1 / 2\right)$ with $N_{A}=0.2, N_{B}=0.1$ in Fig. $7 \mathrm{a}$ and $N_{A}=0.1, N_{B}=0.2$ in Fig. $7 \mathrm{~b}$. The higher strain hardening of the weaker central layer (Fig. 7a) is only able to suppress the short wavelength mode if $\sigma_{A} / \sigma_{B} \geq 1 / 2$ (approximately). When the weaker, higher strain hardening material forms the outer layers it is more effective at suppressing the short wavelength modes but it also lowers somewhat the bifurcation strain associated with the intermediate wavelengths. Although these results are limited, it appears that the higher straining hardening layers are not particularly effective at suppressing short wavelength modes in a power-law multilayer unless their strength is relatively high.

\section{Multilayers with alternating layers of power-law material and neo-Hookean material}

Single layers of the neo-Hookean material do not neck and thus it is natural to ask whether advantage of this property can be exploited to postpone necking in metal/elastomer multilayers. Bilayers comprised of a metal layer bonded to a relatively thick elastomer substrate have been considered in [11] to elucidate enhanced straining in the thin metal film prior to a necking failure with application to stretchable electronics. Theoretical studies and experimental observation of the tearing behavior of metal/polymer multilayers have been carried out which reveal short wavelength necking localization in the metal layers as well as the final fracture process [12]. The potential of a bilayer with comparable thicknesses of metal and elastomer for enhancing energy absorption was investigated in [13] in connection with the localization resistance of structural plates to in-plane stretch. Here the focus is on whether a multilayer with

alternating layers of metal, represented by the power-law material, and elastomer, represented by the neo-Hookean material, is likely to localize in a long wavelength mode associated with the Considère strain or in a short wavelength mode that scales with the thickness of the metal layer. To limit the scope of the study, attention will be focused on systems whose layers have 
comparable thickness rather than the case of thin metal films on thick elastomer substrates explored in [11].

As noted in [11], in plane strain tension the Considère strain, $\varepsilon_{C}$, from the maximum load condition (14) depends on the strain hardening exponent of the metal, $N$, and the single dimensionless parameter

$$
S=\frac{E_{n H} h_{n H}}{\sigma_{0} h_{P L}}
$$

where $E_{n H} \equiv 4 \mu_{0}$ is the ground state plane strain tensile modulus defining the neo-Hookean material (4), $h_{n H}$ is the net thickness of the neo-Hookean layers, $\sigma_{0}$ is the strength of the metal in (3) and $h_{P L}$ is the net thickness of the power-law layers. The ratio $h_{n H} / h_{P L}$ is independent of plane strain stretch. The condition for attaining the Considère strain is

$$
\varepsilon^{N-1}(\varepsilon-N)=S\left(e^{2 \varepsilon}+3 e^{-2 \varepsilon}\right) / 4
$$

and a plot of the Considère strain is given in Fig. 8.

As the first example, consider a 3-layer sheet with a neo-Hookean layer sandwiched between two equal thickness layers of power-law material with $N=0.1$ such that the net thicknesses of the materials are the same, $h_{n H} / h_{P L}=1$, or, in the notation of Fig. $1, h_{2} / h_{1}=1 / 2$. The bifurcation strain for this multilayer is plotted as a function of normalized period, $L$ / $H$, for four values of $E_{n H} / \sigma_{0}$ in Fig. 9a. As defined earlier, $H$ is the total thickness of the multilayer at bifurcation. The Considère strain for each case is indicated as the intercept with the vertical axis on the right hand side of the plot, and this value is approached asymptotically for large $L / H$. For the cases having $E_{n H} / \sigma_{0} \geq 0.2$, the bifurcation strain associated with a short wavelength mode, $L / H \cong 2$, is lower than the corresponding Considère strain. An example of the short wavelength mode shape is presented in Fig. 9b. The critical mode is an anti-symmetric. The thinnest regions of the metal layers in this mode are not aligned vertically but, instead, as noted before are offset in the lateral direction by a half-period. The deformation in the 
bifurcation mode within the central neo-Hookean layer is dominated by shear-the metal layers neck and the elastomer resists by shearing.

Although perhaps of less practical interest, the companion example to that above is a 3layer sheet having a metal layer sandwiched between two neo-Hookean layers, with $h_{2} / h_{1}=1$ / 2 and the same combination of material properties. The bifurcation strain is plotted in Fig. 10. Again, for $E_{n H} / \sigma_{0} \geq 0.2$, the lowest bifurcation strain is lower than the Considère strain. The lowest bifurcation occurs as symmetric mode with $L / H \cong 2$ (not shown) in which the central power-law layer undergoes the periodic thinning similar to that of a single layer of power-law material. The limit in Fig. 10 for $E_{n H} / \sigma_{0} \rightarrow 0$ is the result for a single power-law layer with current thickness $H=h_{1}$.

An example for a 5-layer sheet serves to emphasize that the behavior noted above is not anomalous and that inclined short wavelength localizations are likely to be the rule rather than the exception when the thickness of the metal layers roughly balances that of the elastomer layers, i.e., $h_{n H} / h_{P L} \sim 1$. Fig. 11 shows the bifurcation strain for a 5-layer sheet with the powerlaw material comprising the central layer and the two outermost layers separated by two neoHookean layers. The net thicknesses of the two materials have, $h_{n H} / h_{P L}=1$, with further details specified in the figure. Now, if $E_{n H} / \sigma_{0} \geq 0.2$, the lowest bifurcation strain is associated with a symmetric short wavelength mode having $L / H \cong 1$ (c.f., Fig. 11b). Note, however, that this mode again reveals that the thinnest region of each power-law layer is shifted by a half-period from its neighboring power-law layer such that localization is expected to occur in an inclined band whose lateral extent will be set by the thickness of the individual power-law layer.

As noted in the Introduction, tearing resistance of a multilayer is significantly enhanced if the lateral extent of the necking zone is set by the total thickness of the multilayer rather than the thickness of an individual layer. Thus, in connection with the results discussed above, to achieve high tearing resistance one is led to consider designs that drive the bifurcation strains of the short wavelength mode above those of the long wavelength modes, i.e., above the Considère strain. For a given number of layers and a fixed ratio, $E_{n H} / \sigma_{0}$, this can be achieved by decreasing the relative thickness of the neo-Hookean layers, e.g., decreasing $h_{n H} / h_{P L}$. An example which 
illustrates the transition from short to long wavelength bifurcation is presented in Fig. 12 for a 5layer multilayer. Curves for four values of $h_{n H} / h_{P L}$ are plotted all with $E_{n H} / \sigma_{0}=0.4$.

Decreasing $h_{n H} / h_{P L}$ lowers the Considère strain (c.f., Fig. 8), as revealed by the right-hand intercepts in Fig. 12. The curve for $h_{n H} / h_{P L}=1$ is repeated from Fig. 11 and it shows that the lowest bifurcation strain associated with a mode having with $L / H \cong 1$ is substantially below the Considère strain. The lowest bifurcation strain for multilayers with $h_{n H} / h_{P L}<1 / 2$ is the long wavelength Considère strain. For $h_{n H} / h_{P L}=1 / 8 \& 1 / 4$ the separation between the bifurcation strains associated with the short wavelength modes and the Considère strain is considerable. Thus, a reduction of the relative thickness of the neo-Hookean layers results in the tradeoff between a lowered Considère strain and the suppression of short wavelength localization modes.

\subsection{A multilayer with an infinite number of layers}

Further insight into the competition between localized necking and attainment of the Considère strain is gained extending the considerations above to an infinitely thick multilayer with an infinite number of layers with the power-law material alternating with the neo-Hookean material. The notation remains the same. The period of the layering in the vertical direction is $h_{P L}+h_{n H}$ and the results discussed above suggest that the critical local mode should have a period that is twice $h_{P L}+h_{n H}$. Thus, in searching for the lowest critical mode, four layers are considered (c.f., Fig. 13) and periodic boundary conditions are applied to the bottom and top surfaces. The bifurcation analysis of Section 4 is readily modified to accommodate the periodicity conditions in the vertical direction—details are omitted. The Considère strain is still given by (30).

The three dimensionless parameters fully characterizing the multilayer are $\left(E_{n H} / \sigma_{0}, h_{n H} / h_{P L}, N\right)$. A map showing the regions of the parameter space in which the first bifurcation occurs as a localized mode at a strain less than the Considère strain, $\varepsilon_{C}$, is shown in Fig. 13a. Over the range of $E_{n H} / \sigma_{0}$ plotted, the transition boundary between the two regimes is almost independent of the strain hardening exponent of the power-law material. As noted in connection with the previous example, localized necking can be delayed relative to the Considère strain by diminishing the thickness of the neo-Hookean layers. An example of a bifurcation 
mode shape is shown in Fig. 13b. This mode has a period $2\left(h_{P L}+h_{n H}\right)$ in the vertical direction, rather than $h_{P L}+h_{n H}$ which is also in competition. The characteristic of this mode is again seen to have thinning regions of the power-law layers shifted from one another by one halfwavelength in the horizontal direction. The neo-Hookean layers display bending-like behavior with relatively little change in thickness along their length.

Critical modes having double the wavelength of the periodic microstructure have been found for in-plane compressive buckling of elastic materials with a doubly periodic array of cylindrical voids $[15,16]$. The analysis approach in [15] is more general than the simplified method adopted here for the infinite multilayer in that it allows for arbitrary periodicity. Although we cannot say for certain that the lowest bifurcation strain will have a mode with twice the layering periodicity, the behavior seen for the finite multilayers suggests that it will. In addition, as a check, we repeated the analysis used to generate the results in Fig. 13 by using 8 layers, rather than 4 layers, with no change in the prediction of the lowest bifurcation strain and mode. Thus, critical modes with period $4\left(h_{P L}+h_{n H}\right)$ can be ruled out.

\section{Discussion}

When the lowest bifurcation strain of a multilayer is associated with a short wavelength mode, with period scaling with an individual layer thickness, the localization process which follows is also likely to result in localized necks that are short compared to the multilayer thickness. Examples for a multilayer comprised of alternating layers of a metal with power-law hardening and a neo-Hookean material can have bifurcations first occurring in the short wavelength mode. When the lowest bifurcation strain is associated with the long wavelength Considère strain, short wavelength modes with bifurcation strains only slightly above the Considère strain may exist, as illustrated by a number of examples in this paper. Then, there is a possibility that the short wavelength mode may play a role in the post-bifurcation localization process resulting in a short wavelength neck. The post-bifurcation localization process leading to well-developed necks requires a fully nonlinear analysis. This writer is unaware of any analytical method that has successfully linked the bifurcation mode to the final necked-down state. To date, only numerical methods, and particularly the finite element method for finite strain plasticity, have been able to resolve the evolution of the neck, e.g., [9,10,11,14]. The full 
evolution of the short wavelength necking of a thin metal film bonded to a thick polymer substrate was studied using finite element methods in [11], and the role of decohesion of the interface between the film and the substrate in the localization processwas also explored [14].

Thus, as the above discussion makes clear, it is necessary to emphasize that bifurcation results such as those in the present paper can only be used to provide insights as to the competition between short wavelength and long wavelength modes in multilayer necking. The bifurcation results also suggest ways to suppress short wavelength modes, such as thinning the neo-Hookean layers between metal layers in a multilayer. Further work exploring the evolution from the bifurcation mode to well-formed necks will be required to establish with certainty the final form of the necking localization. As noted in the Introduction, tearing resistance of a ductile multilayer is directly related to whether the short wavelength mode or the long wavelength mode establishes the fully developed neck.

Acknowledgement This work was supported in part by the DARPA program on Materials with Controlled Microstructural Architecture (MCMA) through a sub-grant from the University of Virginia, and in part by the School of Engineering and Applied Sciences, Harvard University.

\section{References}

[1] Barenblatt, G. I. The mathematical theory of equilibrium cracks in brittle fracture. Adv. Appl. Mech. 1962; 7: 55-129.

[2] Dugdale, D.S. Yielding of steel sheets containing slits. J. Mech. Phys. Solids 1960; 8: 100104.

[3] Nielsen, K.L., and Hutchinson, J.W. Cohesive traction-separation laws for tearing of ductile metal plates. Int. J. Impact Engineering 2012; 48: 15-23.

[4] Hill, R. and Hutchinson, J.W. Bifurcation Phenomena in the Plane Tension Test. J. Mech. Phys. Solids 1975; 23: 239-264.

[5] Biot, M.A. Mechanics of incremental deformation. Wiley 1965, New York, NY.

[6] Hutchinson, J.W. and Neale, K.W. Finite Strain J2 Deformation Theory. in Proceedings of the IUTAM Symposium on Finite Elasticity edited by D. E. Carlson and R. T. Shield, Martinus Nijhoff Publishers, Netherlands 1981: 238-247. 
[7] Hill, R. A general theory of uniqueness and stability in elastic-plastic solids. J. Mech. Phys. Solids 1958; 6: 236-249.

[8] Hutchinson, J.W. Plastic Buckling. Adv. App. Mech. 1974; 14: 67-144.

[9] Needleman, A. A Numerical Study of Necking in Circular Cylindrical Bars. J. Mech. Phys. Solids 1972; 20: 111-127.

[10] Tvergaard, V. and Needleman, A. Analysis of the Cup-Cone Fracture in a Round Tensile Bar. Acta Metall. 1984; 32: 157-169.

[11] Li, T. and Suo, Z. Deformability of thin metal films on elastomer substrates. Int. J. Solid Struct. 2006; 43: 2351-2363.

[12] Andreasson, E., Kao-Walter, S. and Stahle, P. Micro-mechanisms of a laminated packaging material during fracture. Eng. J. Fract. Mech. 2013 in press.

[13] Xue, Z. and Hutchinson, J.W. Neck retardation and enhanced energy absorption in metalelastomer bilayers. Mech. of Mater. 2007: 39: 473-487.

[14] Li, T. and Suo, Z. Ductility of thin metal films on polymer substrates modulated by interfacial adhesion. Int. J. Solid. Struct. 2007; 44, 1696-1705.

[15] Triantafyllidis, N., Nestorovic, M.D. and Schraad, M.W. Failure surfaces for finitely strained two-phase periodic solids under general in-plane loading. J. Appl. Mech. 2006; 73, 505515.

[16] Bertoldi, K., Boyce, M.C., Deschanel, S., Prange, S.M. and Mullin, T. Mechanics of deformation-triggered pattern transformations and superelastic behavior in periodic elastomer structures. J. Mech. Phys. Solids 2008; 56, 2642-2668.

\section{Appendix: The matrices $B$ and $T$}

For any layer denote its thickness by $h$, let $w=2 \pi / L$, and recall the definitions $p=\left(E_{t}-\mu-\sigma / 2\right)$ and $q=(\mu-\sigma / 2)$. For $R>0$, with $s a=\sinh (w \alpha h), c a=\cosh (w \alpha h)$, $s b=\sinh (w \beta h)$ and $c b=\cosh (w \beta h)$, 


$$
\begin{aligned}
& B=\left[\begin{array}{cccc}
1 & 0 & 1 & 0 \\
0 & w \alpha & 0 & w \beta \\
0 & w^{3}\left(-p \alpha+q \alpha^{3}\right) & 0 & w^{3}\left(-p \beta+q \beta^{3}\right) \\
-w^{2} q\left(1+\alpha^{2}\right) & 0 & -w^{2} q\left(1+\beta^{2}\right) & 0
\end{array}\right] \\
& T=\left[\begin{array}{cccc}
c a & s a & s b \\
w \alpha s a & w \alpha c a & w \beta s b & w \beta c b \\
w^{3} \alpha s a\left(-p+q \alpha^{2}\right) & w^{3} \alpha c a\left(-p+q \alpha^{2}\right) & w^{3} \beta s b\left(-p+q \beta^{2}\right) & w^{3} \alpha c b\left(-p+q \beta^{2}\right) \\
-w^{2} q c a\left(1+\alpha^{2}\right) & -w^{2} q s a\left(1+\alpha^{2}\right) & -w^{2} q c b\left(1+\beta^{2}\right) & -w^{2} q s b\left(1+\beta^{2}\right)
\end{array}\right]
\end{aligned}
$$

For $R<0$, with $s h=\sinh (w \alpha h), c h=\cosh (w \alpha h), s b=\sin (w \beta h)$ and $c b=\cos (w \beta h)$,

$$
\begin{aligned}
& B=\left[\begin{array}{cccc}
1 & 0 & 0 & 0 \\
0 & w \beta & 0 & w \alpha \\
0 & w^{3} \beta(-p & 0 & w^{3} \alpha(-p \\
-w^{2} q(1 & \left.+q\left(3 \alpha^{2}-\beta^{2}\right)\right) & & \left.+q\left(\alpha^{2}-3 \beta^{2}\right)\right) \\
\left.+\alpha^{2}-\beta^{2}\right) & 0 & -w^{2} q 2 \alpha \beta & 0
\end{array}\right] \\
& T=\left[\begin{array}{cccc}
c h c b & c h s b & \text { shsb } & \text { shcb } \\
w(\alpha \text { shcb }-\beta c h s b) & w(\alpha \text { shs } b+\beta c h c b) & w(\alpha c h s b+\beta \text { shcb }) & w(\alpha c h c b-\beta \text { shs }) \\
w^{3}[-p \alpha(\alpha \text { shcb }-\beta c h s b) & w^{3}[-p \alpha(\alpha s h s b+\beta c h c b) & w^{3}[-p \alpha(\alpha c h s b+\beta s h c b) & w^{3}[-p \alpha(\alpha c h c b-\beta s h s b) \\
+q\left(\alpha\left(\alpha^{2}-3 \beta^{2}\right) s h c b\right. & +q\left(\alpha\left(\alpha^{2}-3 \beta^{2}\right) s h s b\right. & +q\left(\alpha\left(\alpha^{2}-3 \beta^{2}\right) c h s b\right. & +q\left(\alpha\left(\alpha^{2}-3 \beta^{2}\right) c h c b\right. \\
\left.\left.-\beta\left(3 \alpha^{2}-\beta^{2}\right) c h s b\right)\right] & \left.\left.+\beta\left(3 \alpha^{2}-\beta^{2}\right) c h c b\right)\right] & \left.\left.+\beta\left(3 \alpha^{2}-\beta^{2}\right) s h c b\right)\right] & \left.\left.-\beta\left(3 \alpha^{2}-\beta^{2}\right) s h s b\right)\right] \\
-w^{2} q\left[\left(1+\alpha^{2}-\beta^{2}\right) c h c b\right. & -w^{2} q\left[\left(1+\alpha^{2}-\beta^{2}\right) c h s b\right. & -w^{2} q\left[\left(1+\alpha^{2}-\beta^{2}\right) s h s b\right. & -w^{2} q\left[\left(1+\alpha^{2}-\beta^{2}\right) s h c b\right. \\
-2 \alpha \beta \text { shsb }] & +2 \alpha \beta s h c b] & +2 \alpha \beta c h c b] & -2 \alpha \beta c h s b]
\end{array}\right]
\end{aligned}
$$




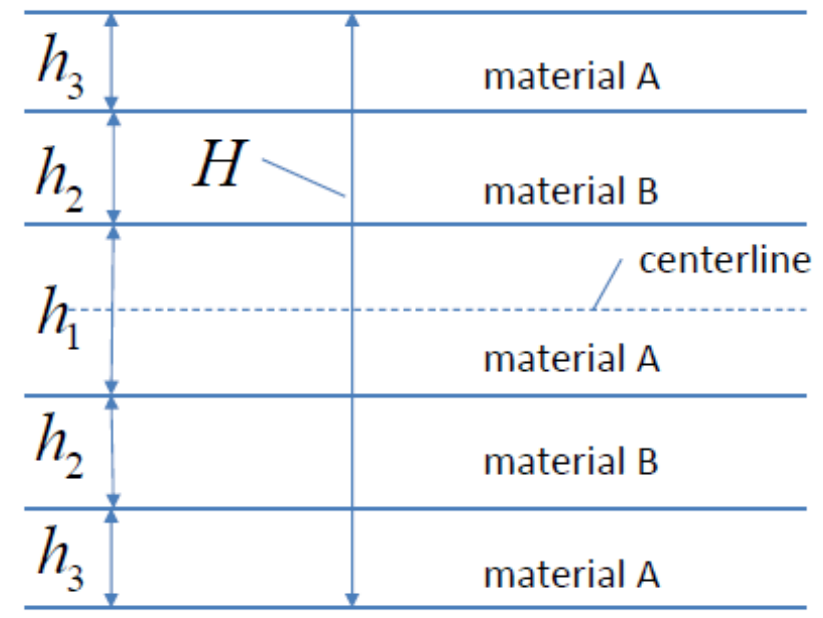

Fig. 1 Symmetric multilayer with 2M-1 layers ( $M=3$ in the above). Material B alternates with material A with material A at the center.

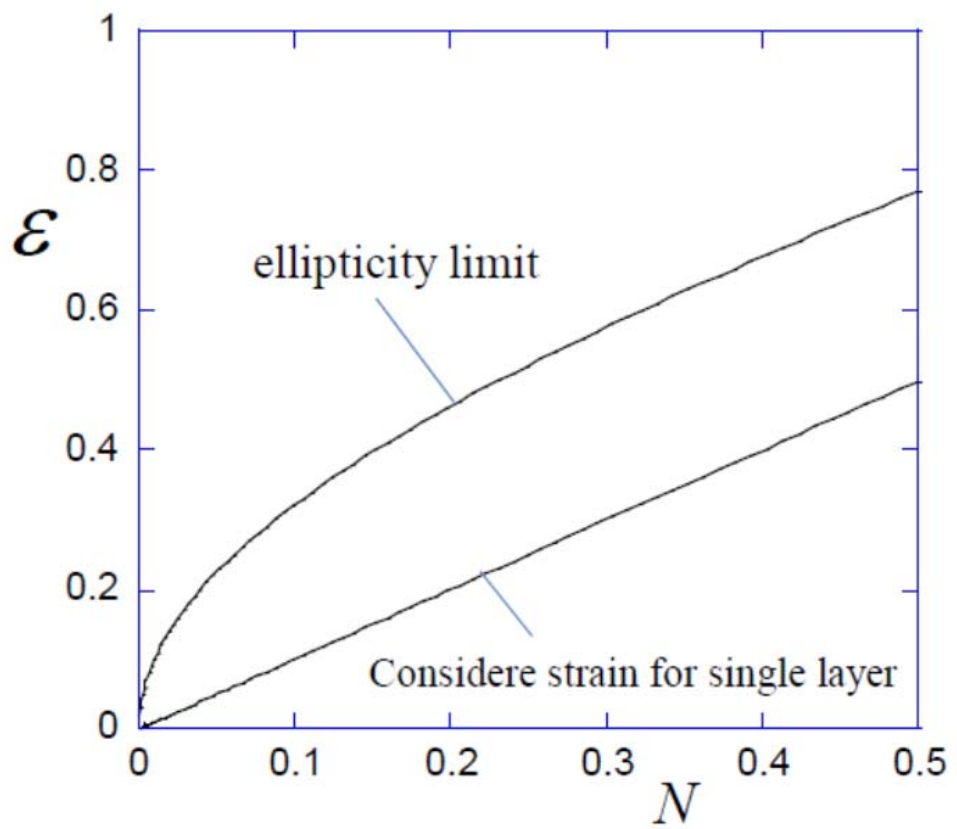

Fig. 2 Ellipticity limit in plane strain tension for the power-law material. The Considère strain for a free-standing single layer of the material is also shown. 

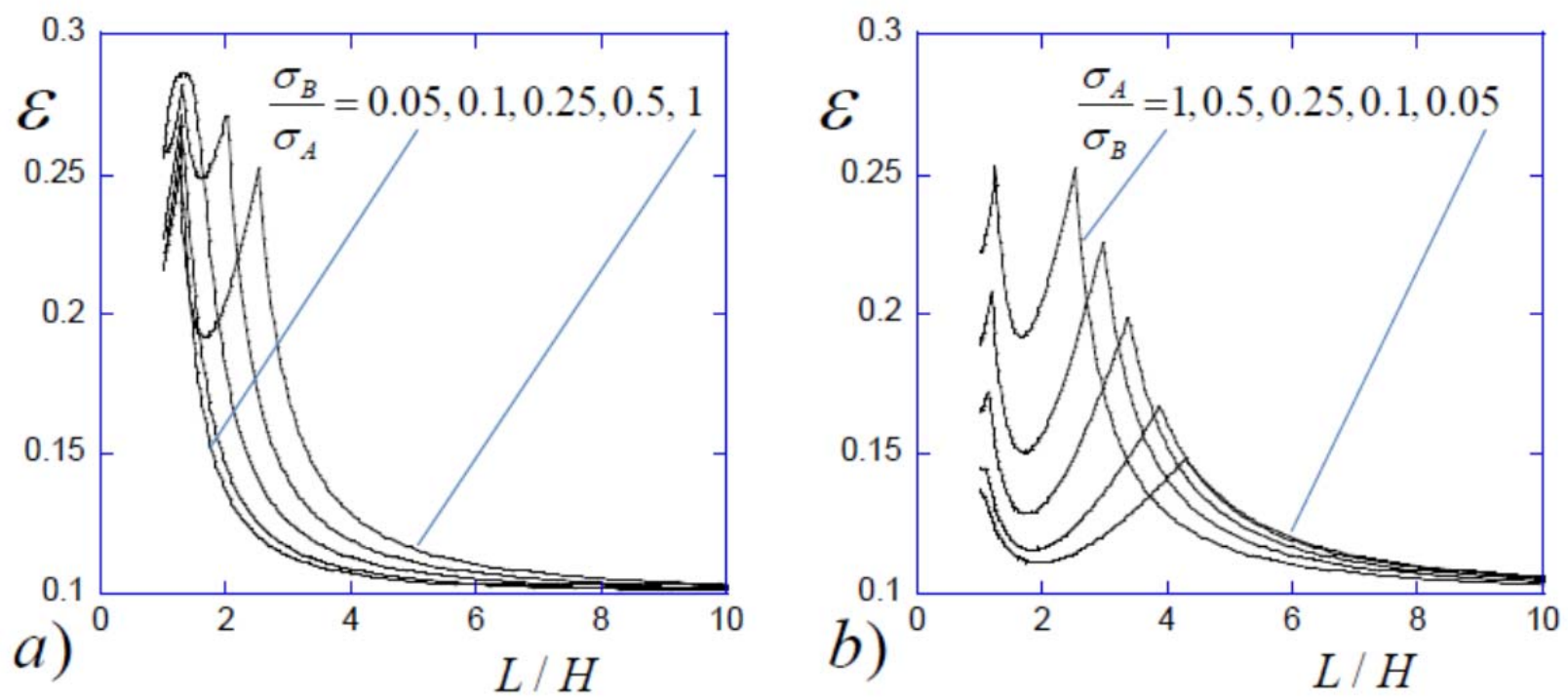

Fig. 3 Bifurcation strain as a function of the normalized wavelength for a power-law multilayer having 3-layers with material A as the central layer and material B as the two outer layers. $N_{A}=N_{B}=0.1$ and $h_{B} / h_{A}=1\left(h_{2} / h_{1}=1 / 2\right)$. a) The stronger material in the central layer. b) The weaker material in the central layer.

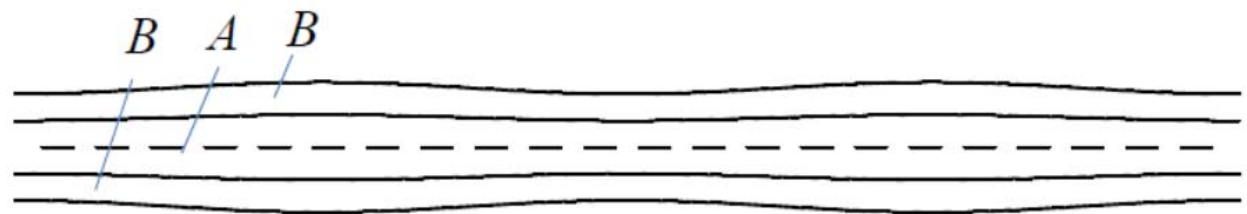

$L / H=10$, symmetric mode

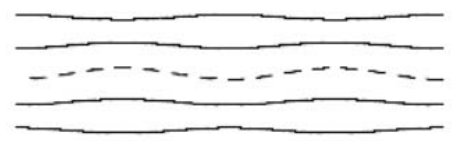

$L / H=2$, anti-symmetric mode

Fig. 4 The bifurcation mode shapes associated with Fig. $3 \mathrm{~b}$ for the case of the weaker material in the central layer ( $\sigma_{0}^{A} / \sigma_{0}^{B}=0.05$ ). The plots display the variation of the normal displacement increment, $v_{2}$, for the layer boundaries (and the centerline, which is dashed) associated with the bifurcation mode. The sign and amplitude of the shape are arbitrary. 

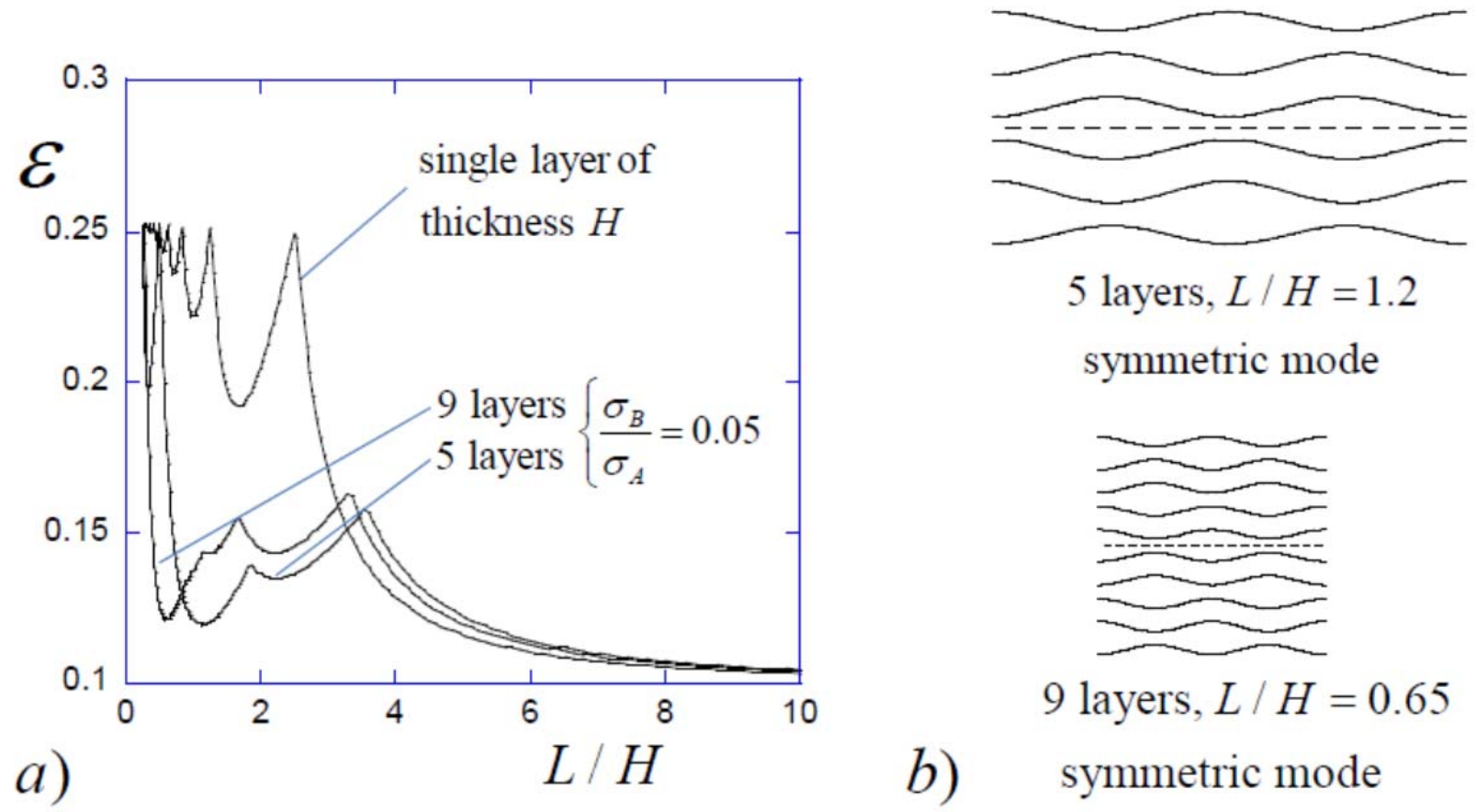

5 layers, $L / H=1.2$

symmetric mode

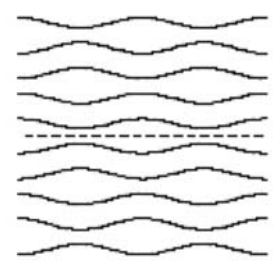

9 layers, $L / H=0.65$

b)

symmetric mode

Fig. 5 The influence of the number of layers on the bifurcation strain a) and the mode shape b). $N_{A}=N_{B}=0.1$. The result for a single layer (of either material) is included for reference. For the 5-layer and 9-layer cases the thickness of all the layers is the same and the stronger material (A) is in the central layer (and in the outermost layers). Layers of material B alternate with layers of material A. The mode shapes reflect the fact that the thinning regions of neighboring strong layers are shifted by a half-wavelength. 


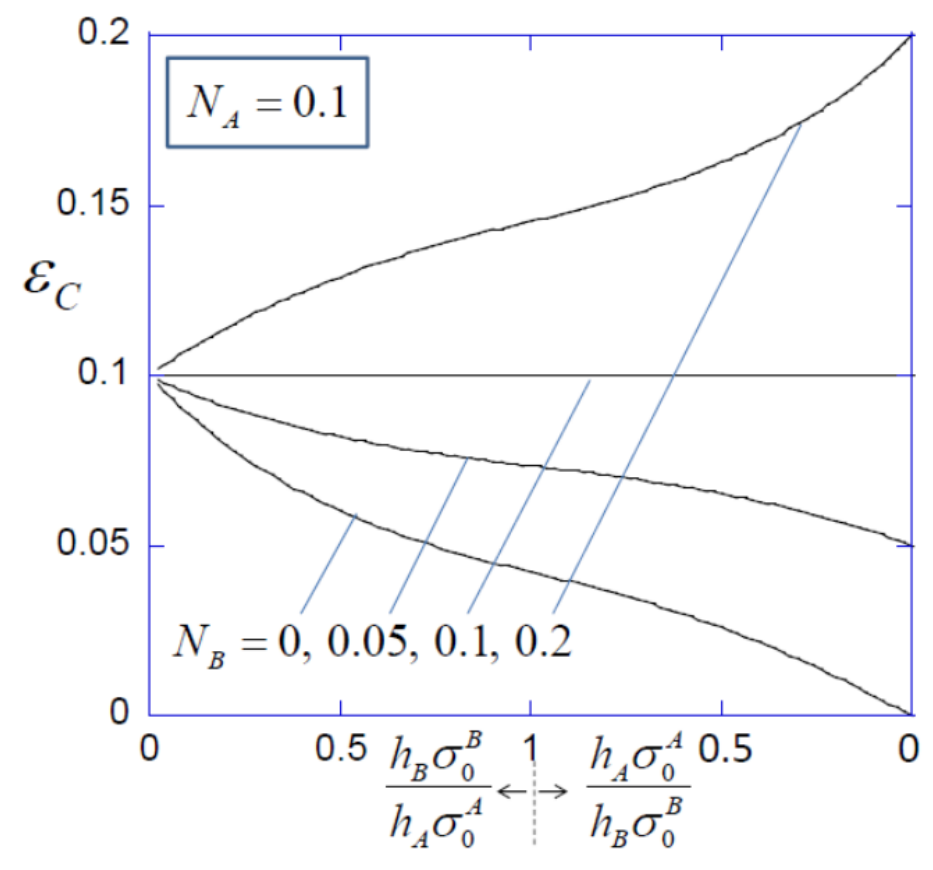

Fig. 6 The Considère strain for a multilayer of two power-law materials.
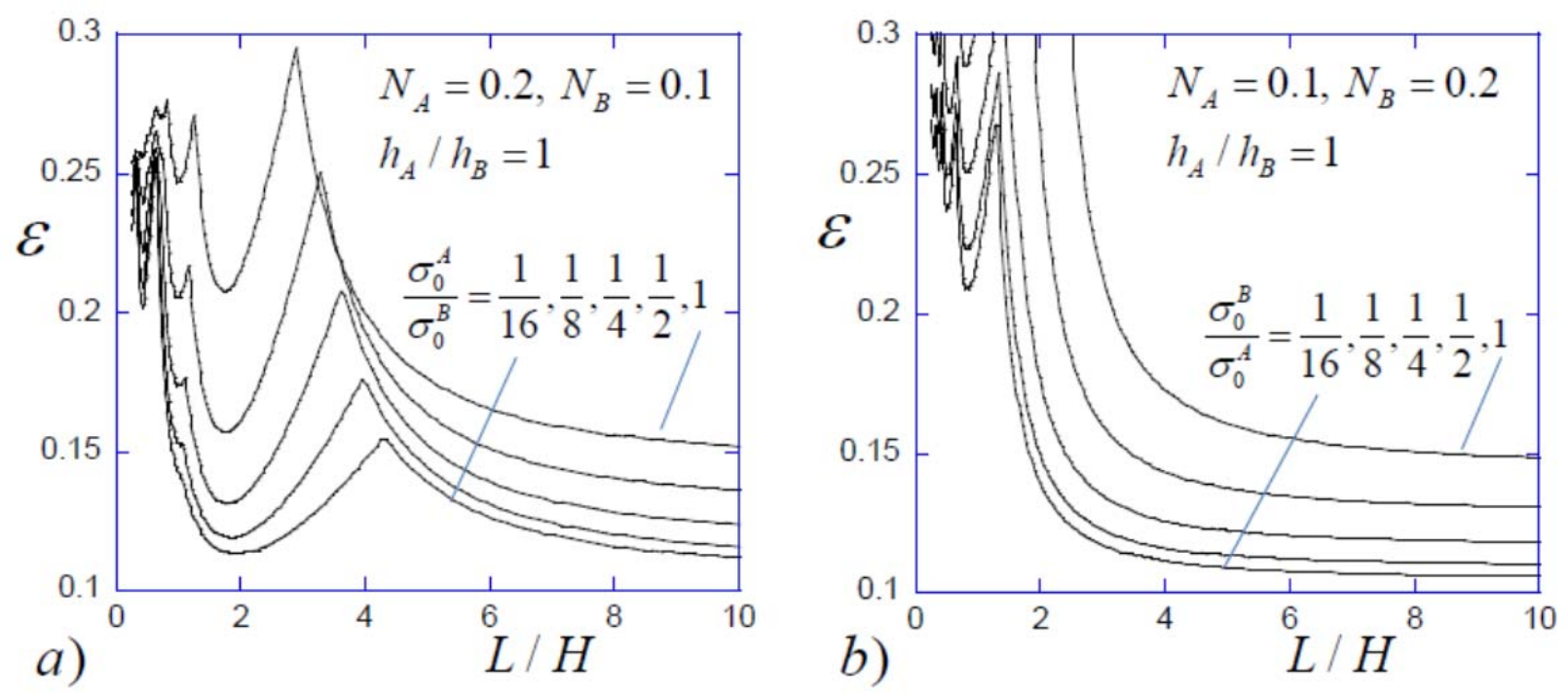

Fig. 7 The bifurcation strain as a function of normalized wavelength for a 3-layer multilayer comprised of power law materials having different hardening exponents. In a), the weaker material with the higher strain hardening is in the central layer. In b), the stronger material with the lower strain hardening is in the central layer. 


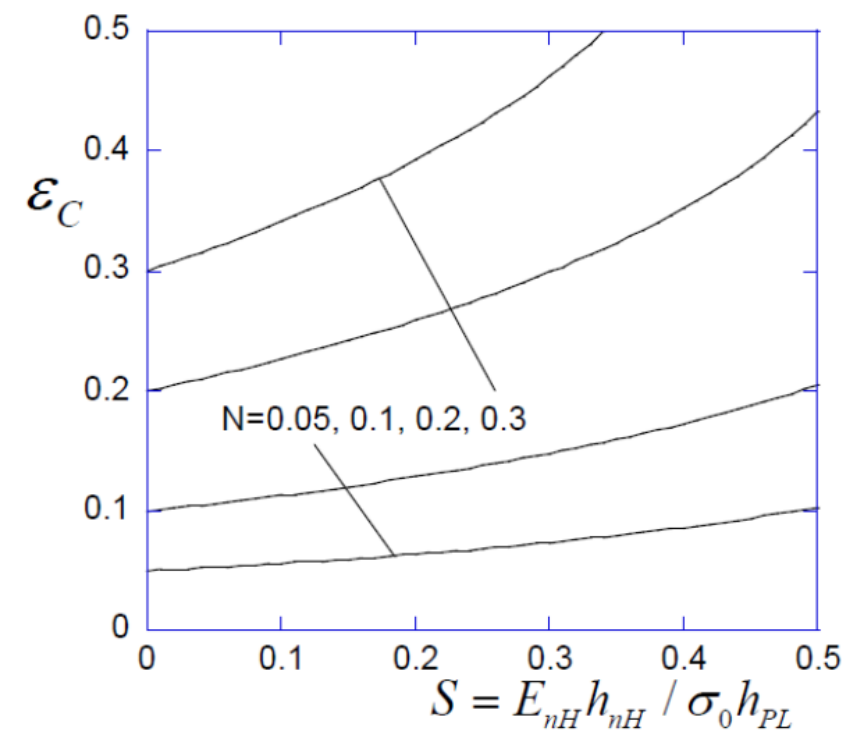

Fig. 8 The Considère strain for a multilayer combining a power-law material and a neoHookean material.

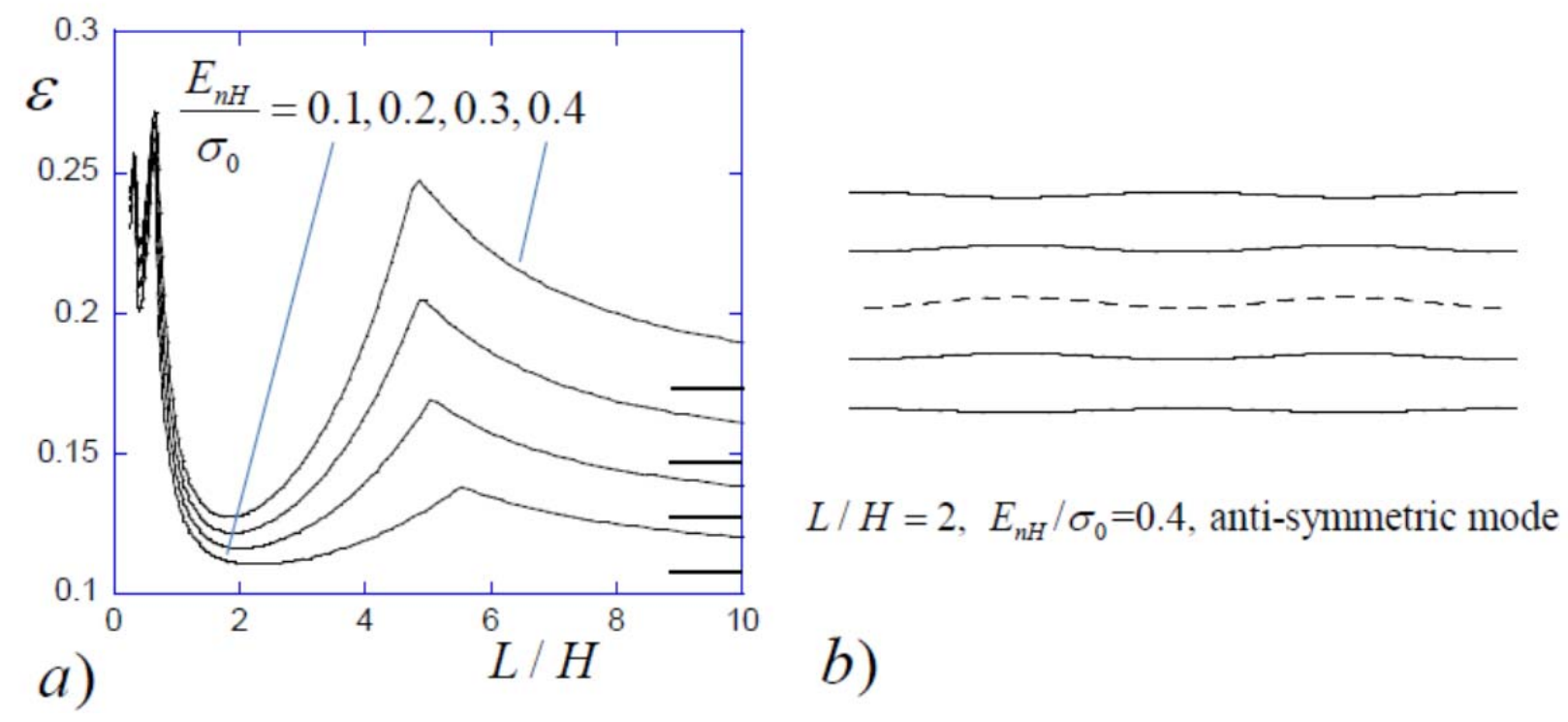

Fig. 9 The bifurcation strain a) and a mode shape b) of a 3-layer multilayer with a neo-Hookean central layer and a power-law material in the outer layers; $N=0.1, h_{B} / h_{A}=1\left(h_{2} / h_{1}=1 / 2\right)$. The Considère strain for the long wavelength limit is indicated by the horizontal markers on the right. A short wavelength mode has the lowest bifurcation strain if $E_{n H} / \sigma_{0} \geq 0.2$. The thinnest regions in the outer layers in short wavelength mode in b) are shifted from one another by a half wavelength. 


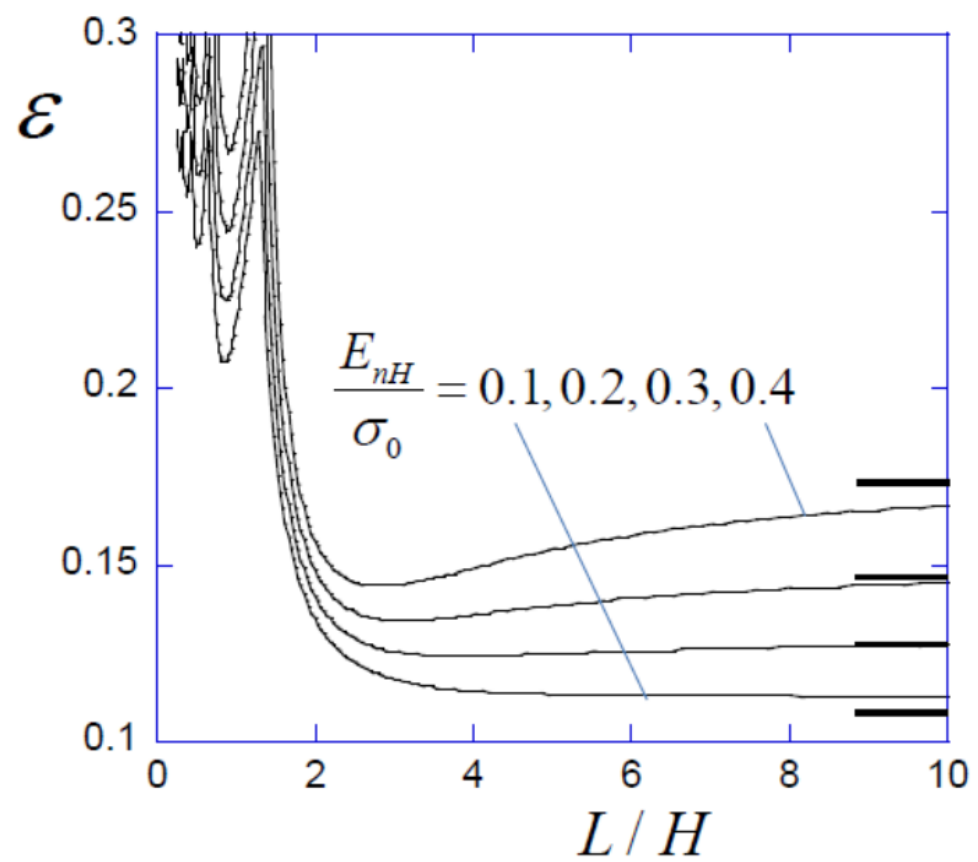

Fig. 10 The companion results to those in Fig. 9. In this case, the 3-layer multilayer has the neoHookean material in the outer layers and the power-law material in the central layer. Otherwise, the parameter choices are the same. The Considère strain for the long wavelength limit is indicated by the horizontal markers on the right. 


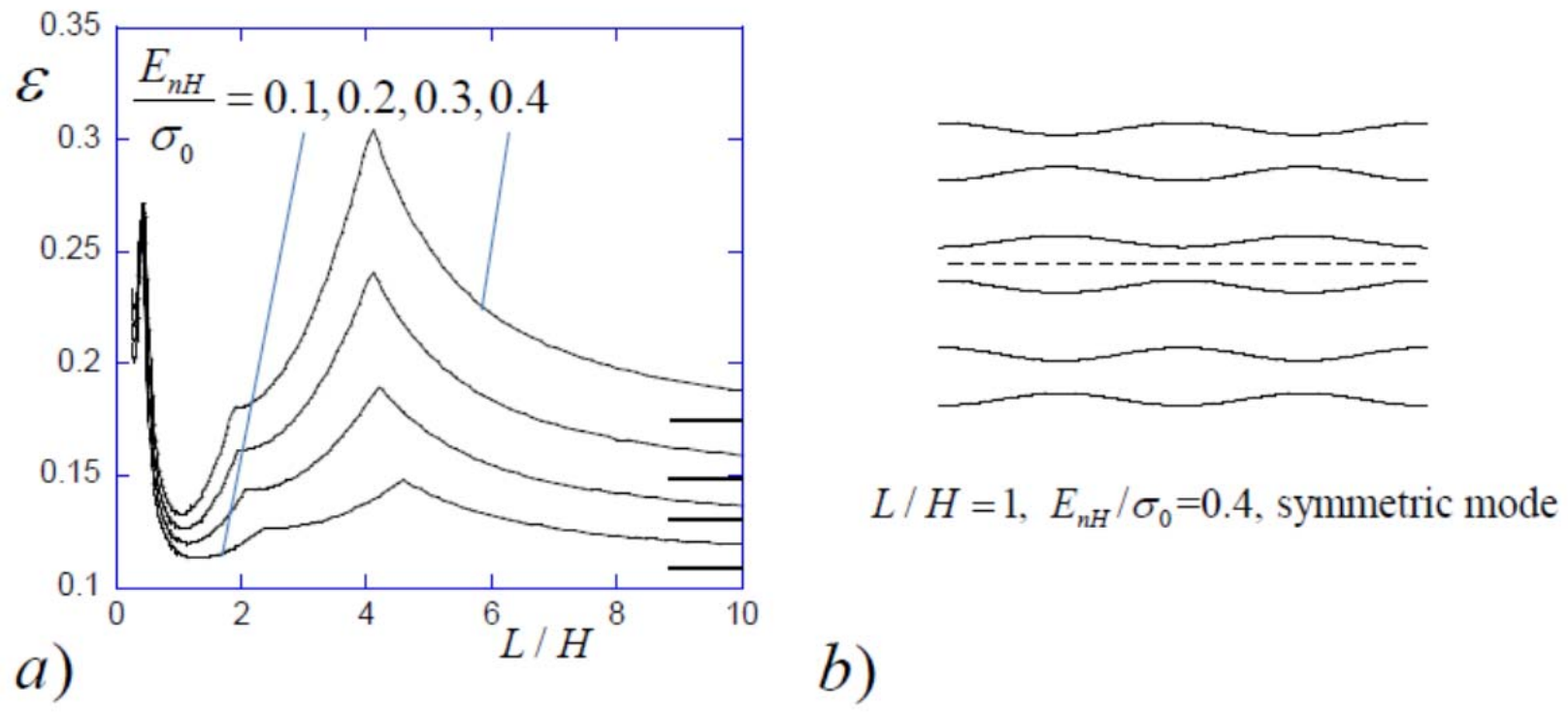

Fig. 11 The bifurcation strain a) and a mode shape b) for a 5-layer multilayer having the powerlaw material ( $N=0.1$ ) in the central and outermost layers with the neo-Hookean material sandwiched between. $h_{B} / h_{A}=1\left(h_{1} / h_{2}=h_{3} / h_{2}=2 / 3\right)$. 


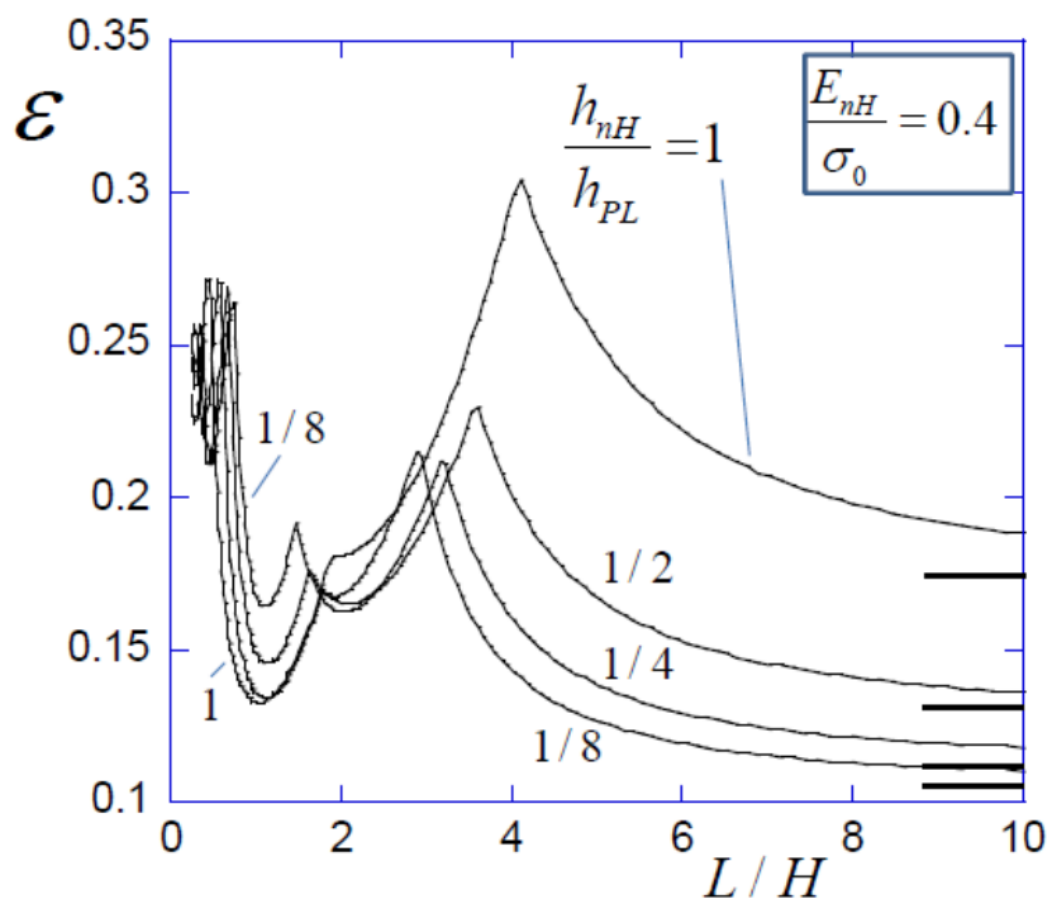

Fig. 12 The effect of decreasing the relative thickness of the neo-Hookean layers in suppressing the short wavelength bifurcation mode for a 5-layer multilayer. The central layer and the outermost layers are the power-law material ( $N=0.1$ ); the two layers separating them are neoHookean. The thickness of each of the three power-law layers are the same, as is the thickness of the two neo-Hookean layers. Four cases are chosen showing the effect of decreasing the relative thickness of the neo-Hookean layers, correspond to $h_{n H} / h_{P L}=1,1 / 2,1 / 4$ and $1 / 8$. The Considère strain for the long wavelength limit is indicated by the horizontal markers on the right. 

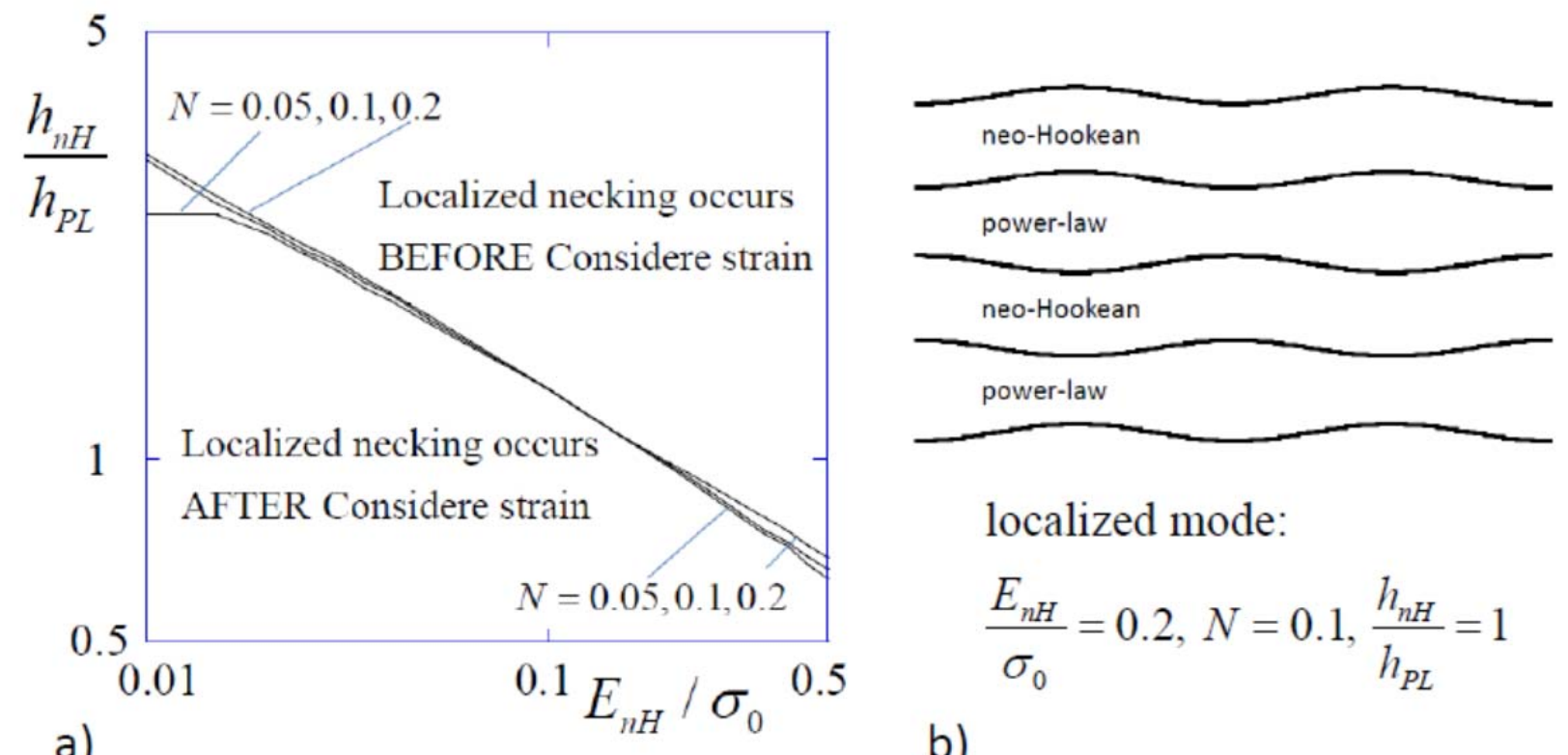

localized mode:

$\frac{E_{n H}}{\sigma_{0}}=0.2, N=0.1, \frac{h_{n H}}{h_{P L}}=1$

b)

Fig. 13 Results for a multilayer with an infinite number of layers with layers of a power law material $\left(h_{P L}, \sigma_{0}, N\right)$ alternating with layers of a neo-Hookean material $\left(h_{n H}, E_{n H}\right)$. a) A map showing whether localized necking occurs before or after attainment of the Considère strain in the dimensionless parameter space. b) An example of the critical local mode for which $L / h_{P L}=5.6$. The mode is periodic in both the horizontal and the vertical directions. Two wavelengths are plotted in the horizontal direction and one wavelength in the vertical direction. 